\title{
Microstructural Evaluation of Magnetocaloric Ni-Co-Mn-Sn Produced by Directed Energy Deposition
}

Erica Stevens ${ }^{1}$, Jakub Toman ${ }^{1}$, Katarina Kimes ${ }^{1}$, Volodymyr Chernenko ${ }^{2,3}$, Anna Wojcik ${ }^{4}$, Wojciech Maziarz $^{4}$, and Markus Chmielus ${ }^{1}$

1. University of Pittsburgh, Materials Science and Engineering, Pittsburgh, PA USA

2. Basque Center for Materials, Applications, and Nanostructures, University of Basque Country (UPV/EHU), Bilbao, Spain

3. Ikerbasque, Basque Foundation for Science, Bilbao, Spain

4. Polish Academy of Science, Institute of Metallurgy and Materials Science, Krakow, Poland

Magnetocaloric (MC) materials - materials that experience temperature changes due to applied magnetic fields - have the potential to transform refrigeration and cooling by reducing energy consumption of commercial refrigerators by up to $30 \%$ [1]. Some Heusler alloys (ferromagnetic alloys containing no ferromagnetic elements) including $\mathrm{Ni}-\mathrm{Mn}-\mathrm{Sn}$ with Co addition, are important as MC material systems because they do not require rare earth metals. Ni-Co-Mn-Sn experiences a first-order phase transformation from martensite to austenite. When this transformation (which affects magnetic properties) coincides with the Curie temperature, a MC effect is observed [2]. The spatial freedom of additive manufacturing (AM) would provide the ability to print such materials in a variety of geometries to meet a wider variety of device requirements [3]. As a foundation for combining the benefits of this novel materials system and manufacturing process effectively, the microstructure of AM MC materials must be characterized.

$\mathrm{Ni}_{43} \mathrm{Co}_{7} \mathrm{Mn}_{39} \mathrm{Sn}_{11}$ powder was produced by melt spinning and subsequent grinding by mortar and pestle. Powder morphology was evaluated by optical microscopy of mounted and unmounted powder (Figure 1). A large range of powder sizes was observed, with a maximum Feret's diameter ranging from less than 5 $\mu \mathrm{m}$ to around $100 \mu \mathrm{m}$. Many particles had a rectangular cross-section when polished, with aspect ratios between 0.4 and 0.6 .

Directed energy deposition (DED) with a laser energy source was used to produce a 5-layer, single-line sample of $\mathrm{Ni}_{43} \mathrm{Co}_{7} \mathrm{Mn}_{39} \mathrm{Sn}_{11}$ which was subsequently cross sectioned, polished, and heated to $140{ }^{\circ} \mathrm{C}$ to induce a martensitic transformation. The sample was characterized in both as-printed and heated states using a Keyence VHX-600 digital optical microscope (DOM). A JEOL JSM-6510 scanning electron microscope (SEM) equipped with energy-dispersive X-ray spectroscopy (EDS) was used to examine the sample after heating.

As expected from a rapid solidification process like DED, dendrites were observed in the MC AM sample (Figure 2). Preliminary measurements showed secondary dendrite arm spacing to be approximately $5 \mu \mathrm{m}$. SEM analysis revealed that sub-micron twins did appear on heating and occurred in small, localized areas. Backscatter electron imaging (BSE) showed segregation (Figure 2), and EDS was used to determine that there was a region of Sn depletion at the subgrain boundaries (the interdendritic region).

The results indicate that during DED, the high temperature causes the $\mathrm{Sn}$ to be depleted from subgrain boundaries and thus causes a large variation of composition on very small length scale. Thus, composition ranges that support martensite at room temperature seem to be very localized. 
References:

[1] J.F. Scott, Ann. Rev. Mater. Res. 41 (2011).

[2] A.L. Victor et al., J. Appl. Phys. 119 (2016).

[3] S. Mellor, L. Hao, D. Zhang, Int. J. Prod. Econ. 149 (2014) pp. 194-201.

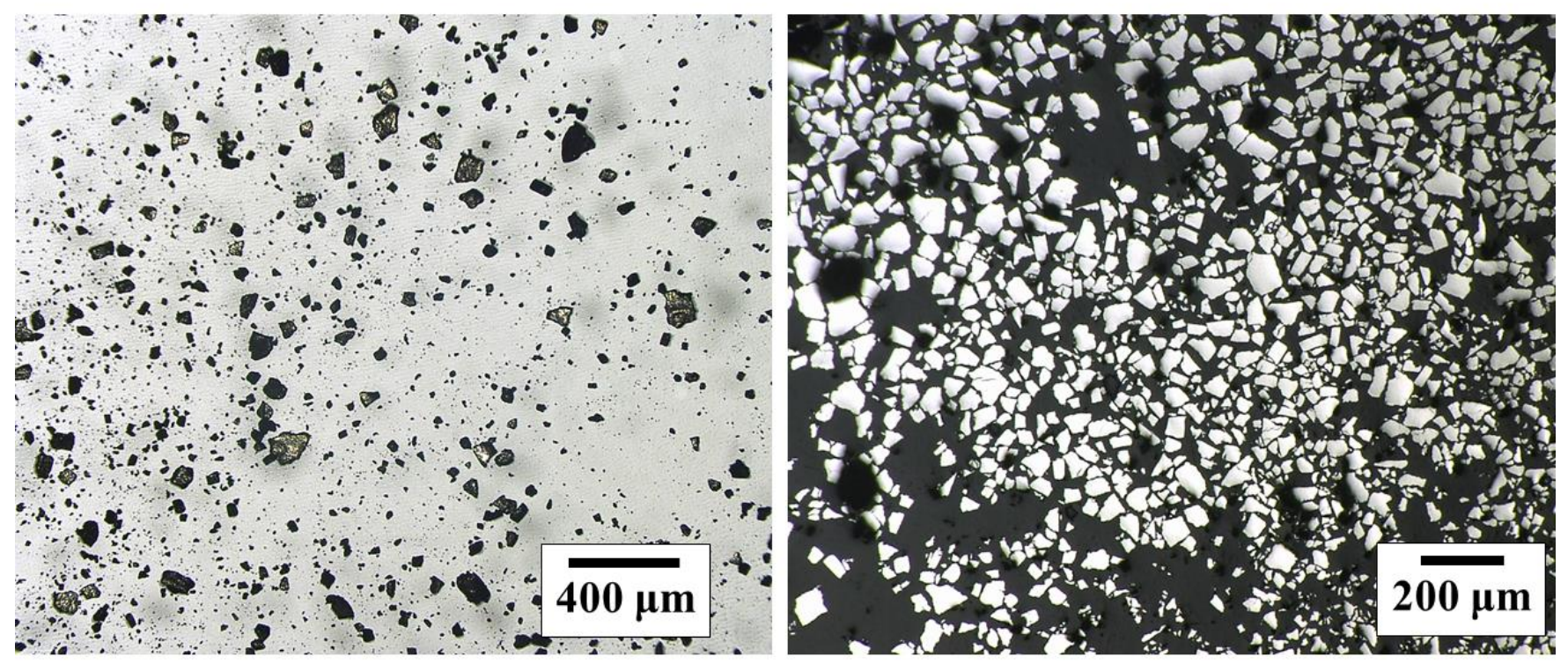

Figure 1. DOM images of $\mathrm{Ni}_{43} \mathrm{Co}_{7} \mathrm{Mn}_{39} \mathrm{Sn}_{11}$ powder produced by melt spinning and subsequent pulverization with mortar and pestle, unmounted at 100x (left) and mounted and polished at 50x (right).

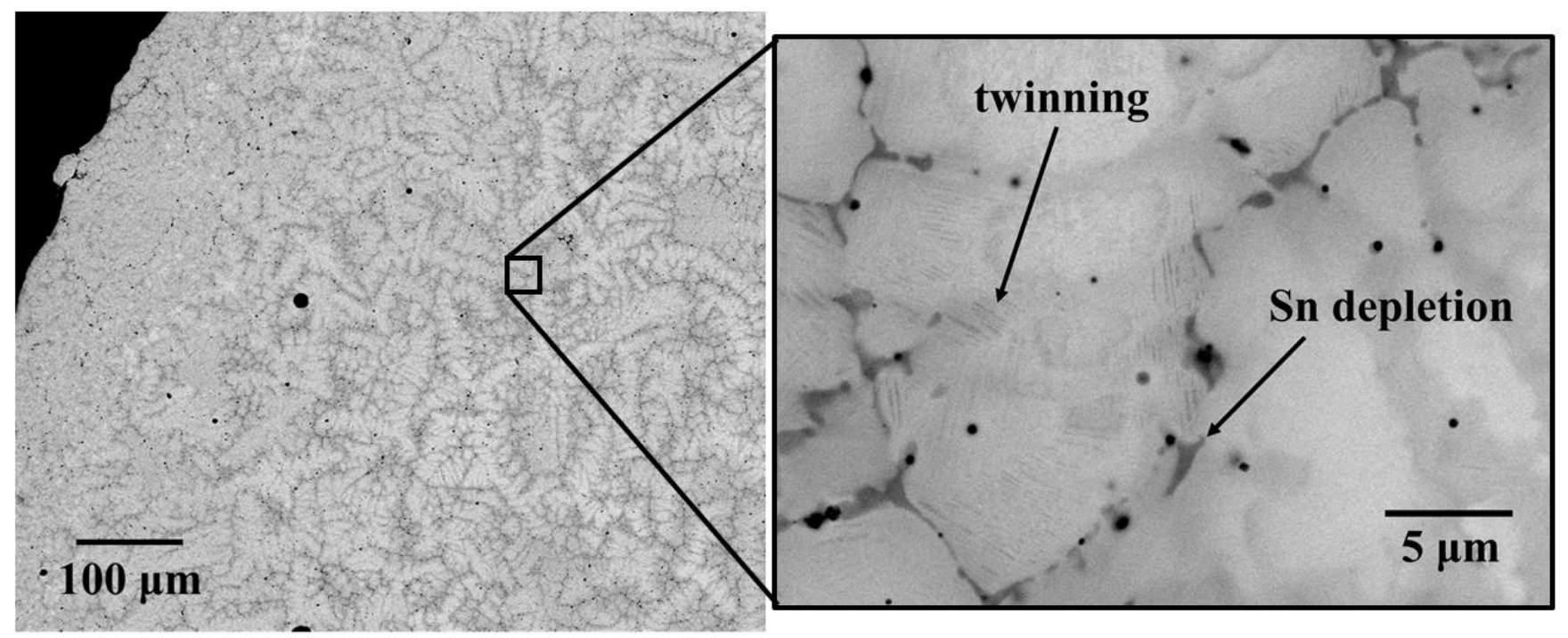

Figure 2. Backscatter electron images at 200x (left) and 4000x (right) with $25 \mathrm{keV}$, showing dendritic structure, twinning, and elemental segregation in AM MC material printed by DED. 